\title{
Three-Dimensional Segmentation of Brain Aneurysms in CTA Using Non-parametric Region-Based Information and Implicit Deformable Models: Method and Evaluation
}

\author{
Monica Hernandez ${ }^{1}$, Alejandro F. Frangi ${ }^{1}$, and Guillermo Sapiro ${ }^{2}$ \\ 1 Computer Vision Group, Division of Biomedical Engineering, Aragon Institute of \\ Engineering Research, University of Zaragoza, Zaragoza, Spain \\ \{mhg, afrangi\}@unizar.es \\ 2 Electrical Engineering and Computer Sciences Department, University of \\ Minnesota, Minneapolis, USA
}

\begin{abstract}
Knowledge of brain aneurysm dimensions is essential in minimally invasive surgical interventions using Guglielmi Detachable Coils. These parameters are obtained in clinical routine using 2D maximum intensity projection images. Automated quantification of the three dimensional structure of aneurysms directly from the 3D data set may be used to provide accurate and objective measurements of the clinically relevant parameters. In this paper we present an algorithm devised for the segmentation of brain aneurysms based on implicit deformable models combined with non-parametric region-based information. This work also presents the evaluation of the method in a clinical data base of 39 cases.
\end{abstract}

\section{Introduction}

Brain aneurysms are pathological dilatations of cerebral arteries. They tend to occurr at or near arterial bifurcations, mostly in the Circle of Willis. Studies assert that the incidence is between 0.2 and $8.9 \%$ with fatal consequences in $2.6-9.8 \%$ and serious consequences in $10.9 \%$ of the patients due to intra cranial bruise, subsequent recurrent bleeding, hydrocephaly and spasms [13. In the last years, there has been a growing trend to practise minimally invasive endovascular procedures. Aneurysm coiling with Guglielmi Detachable Coils (GDC) is probably the most widespread method for permanent aneurysm embolization. The placement of coils inside the aneurysm promotes blood coagulation avoiding blood flow and pressure thus hampering its rupture [6]. A correct size selection and placement of the GDC inside the aneurysm is crucial for the success of the treatment. It has been shown that the knowledge about the dimensions of the aneurysm plays an important role in the selection of patients and materials for an appropriate treatment [3].

To obtain the aneurysm dimensions, it is customary to generate a reconstruction from the CTA images using Maximum Intensity Projection (MIP). Manual measurements are then carried out on the basis of this these $2 \mathrm{D}$ projections. The selection of the window levelling settings of the console and the optimal 
projection angle introduce a high degree of subjectivity to the quantification of the aneurysm morphology. Therefore, the use of computerized 3D segmentation techniques is crucial for an accurate quantification of the aneurysm dimensions as well as a correct interpretation of the 3D morphology.

Most approaches used for the segmentation of vascular structures in CTA and MRA images belong to the class of deformable models [5149]. Although a number of algorithms based on implicit deformable models have addressed the problem of cerebral vessels segmentation [94], these do not produce satisfactory results when confronted with images of standard quality in average radiology departments of hospitals. For example, the work reported by Deschamps [4 deals with rotational angiography where the background and bone tissues have a well differentiated contrast with respect to vessels. On the other hand, the ranges of vessel and bone intensities in CTA overlap. Most of the previous attempts to solve this problem have presented segmentation results on few selected images. There is a general lack of larger evaluation studies on image databases acquired under routine clinical conditions. The Geodesic Active Regions model [1] (GAR) combines the classical Geodesic Active Contours model (GAC) with regional information. This method has been successfully used in the segmentation of brain aneurysms 47. These authors model region-based information by Gaussian descriptors. However, the assumption of a parametric form for the probability density function is not always valid, in particular not in our CTA images.

The goal of this article is to include non-parametric information in the GAR model. This is done using a nearest-neighbor classifier to estimate the underlying probability density functions. The method has been evaluated in a database of 39 brain aneurysms placed within the Circle of Willis. The technique is compared against manual measurements of three geometrical descriptors of aneurysm morphology that are standard in assessing the viability of surgical treatment with GDCs.

The paper is organized as follows. Section 2 explains the devised segmentation method. Section 3 describes the materials and methods used for its evaluation. The results of the study are reported in Section 4. The discussion and conclusion of the results is presented in Section 5 .

\section{Aneurysm Segmentation}

In this article, we propose to use a k-nearest neighbor $(\mathrm{kNN})$ rule to estimate the underlying tissue probability functions for vessel, background and bone. A Maximum A Posteriori (MAP) classifier is used to obtain a rough classification of the image domain into regions matching with each tissue. The likelihood used for each class is the probability estimated by the kNN rule. Then, the GAR method is used to obtain a $3 \mathrm{D}$ model of the aneurysms with subvoxel accuracy. The probabilities estimated by the $\mathrm{kNN}$ rule are also used to build regional descriptors and the voxels belonging to vessel in the MAP partition are used as initialization for an improved GAR method. 


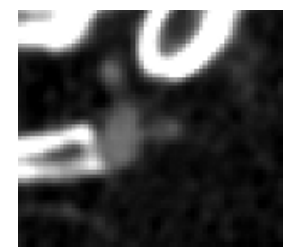

(a)

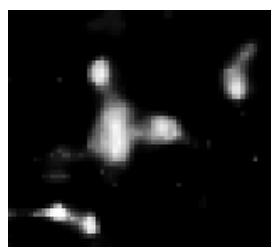

(b)

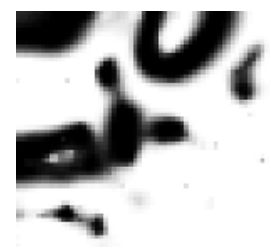

(c)

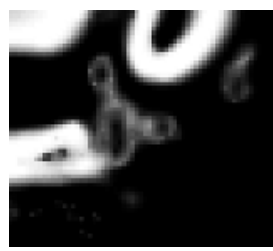

(d)

Fig. 1. Cross-section of the probability function images estimated by the kNN rule. Brighter areas correspond to higher probabilities. (a) Grey level image. (b)-(d) Probability for vessel, background and bone respectively.

\subsection{Non Parametric Tissue Probability Estimation}

The probability function for each tissue is estimated as follows. For the construction of the training set, six images were selected from the data base including the most characteristic patterns in brain aneurysm CTA images. A total of 1830 points were manually picked from the images and labelled with one of these three tissue classes: vessel, background or bone. A label and a feature vector is associated to each point of the training set. This vector is based on the local differential structure of the image at a small scale.

For a point $\hat{\mathbf{x}}$ in the training set, we associate the feature vector $\mathbf{f}(\hat{\mathbf{x}})=$ $\left(I_{\sigma},\left|\nabla I_{\sigma}\right|, \lambda_{1_{\sigma}}, \lambda_{2_{\sigma}}, \lambda_{3_{\sigma}}\right)$ where $I_{\sigma}$ represents the convolution of the image with a Gaussian kernel of $\sigma=0.8 \mathrm{~mm}$. The parameters $\lambda_{i_{\sigma}}$ represent the eigenvalues of the Hessian matrix of the image $I_{\sigma}$, ordered by increasing magnitude.

At this point, the $\mathrm{kNN}$ rule is used to estimate the probability functions as follows. For a given voxel $\mathbf{x}$, the feature vector $\mathbf{f}(\mathbf{x})$ is computed and the $\mathrm{k}$ nearest feature vectors are found in the training set according to the Euclidean distance. Then, the probability for a voxel of intensity $i$ to belong to a tissue class $C_{j}$, is computed from the formula

$$
P\left(I(\mathbf{x})=i \mid C_{j}\right)=\frac{\sum_{\hat{\mathbf{x}} \in \mathcal{L}_{j} \cap \mathcal{N}_{k}(\mathbf{x})} d(\mathbf{f}(\mathbf{x}), \mathbf{f}(\hat{\mathbf{x}}))}{\sum_{\hat{\mathbf{x}} \in \mathcal{N}_{k}(\mathbf{x})} d(\mathbf{f}(\mathbf{x}), \mathbf{f}(\hat{\mathbf{x}}))}
$$

where $\mathcal{L}_{j}$ represents the set of points of the training set that belongs to the class $C_{j}, \mathcal{N}_{k}(\mathbf{x})$ is the set of the $\mathrm{k}$ nearest neighbors and $d$ represents the Euclidean distance. In our approach we used $k=10$. Figure 1 shows an example of the probability functions estimated by the $\mathrm{kNN}$ rule. In the sequel, $C_{0}, C_{1}$ and $C_{2}$ will stand for vessel, background and bone classes respectively.

\subsection{Maximum a Posteriori Tissue Classification}

At this point, a MAP tissue classifier is used to obtain a partition of the image domain into regions matching with vessel, background and bone. The probabilities estimated from the $\mathrm{kNN}$ rule, provide some learned prior probability that 


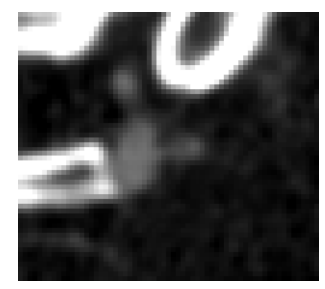

(a)

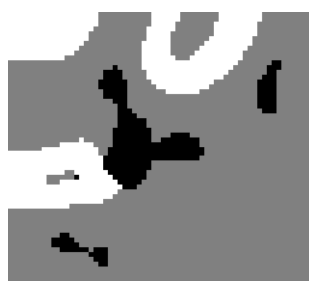

(b)

Fig. 2. Maximum a posteriori classification. (a) Cross-section of the grey level volumetric image. (b) Cross-section with the MAP labels. Black corresponds to vessel tissue, white to bone and grey to background.

a particular voxel belongs to a certain class, $P\left(I(\mathbf{x})=i \mid \mathbf{x} \in C_{j}\right)$. All tissue class are assumed to be equiprobable. The Bayes rule is applied to calculate the posterior probability that a given voxel belongs to a particular class given its intensity, $P\left(C_{j}=c_{j} \mid I(\mathbf{x})=i\right)$. The MAP classifier uses the maximum a posteriori probability estimate after anisotropic smoothing 12 to obtain a classification of the voxels of the image as follows

$$
C_{j}^{*}=\arg \max _{c_{j} \in\left\{C_{0}, C_{1}, C_{2}\right\}} P^{*}\left(C_{j}=c_{j} \mid I(\mathbf{x})=i\right)
$$

where $P^{*}$ corresponds to the posterior probabilities after diffusion. This filter has the property of being grey scale invariance. Therefore, the diffused posteriors remain being probability functions. Applying anisotropic diffusion introduces spatial coherence before the MAP decision by improving the classification results [15]. Figure 2] shows one example of the MAP classification. Voxels labelled as vessel are used as initialization of the GAR method introduced in the next section.

\subsection{Geodesic Active Regions}

The GAR model [11] combines the classical GAC model [2] with region-based statistical information. The model incorporates region-based statistical information into the classical energy functional. Therefore, in places where the gradient is weak, regional information drives the evolution of the front thus being more robust than GAC. Similar work including statistical information on the implicit model was done in [16].

Given a partition of the image domain $\Omega=\Omega_{\text {in }} \bigcup \Omega_{\text {out }}$, the inner region descriptor is defined as $k_{i n}(\mathbf{x})=-\log \left(P_{i n}(\mathbf{x})\right)$, where $P_{\text {in }}$ is the probability that a voxel $\mathbf{x}$ belongs to $\Omega_{i n}$. An analogous definition holds for the outer region. In the work of Paragios [11], region-based information is modelled by time dependent Gaussian descriptors and the partition of the image domain is defined by the front in evolution. However, the assumption of a unimodal parametric form for the probability density function is not always justified in practical applications 
like ours. In our experience, the distributions are not even Gaussian and the intensity distribution of the vessel and the bone are overlapped. For this reason, we propose to use the probabilities learned from the $\mathrm{kNN}$ rule. This probabilities adapt to the distributions of the underlaying tissues and are time- and frontindependent. So $P_{\text {in }}=P\left(I(\mathbf{x})=i \mid C_{0}\right)$ and $P_{\text {out }}=P\left(I(\mathbf{x})=i \mid C_{1}\right)+P(I(\mathbf{x})=$ $\left.i \mid C_{2}\right)$.

The evolution can be expressed by the partial differential equation resulting from the gradient descent flow that minimizes the GAR energy functional $\frac{\partial \Gamma(t)}{\partial t}=\zeta\left(k_{\text {out }}-k_{\text {in }}\right) \vec{N}-\eta(g \kappa+\nabla g \vec{N}) \vec{N}$ with associated level set equation [10]

$$
\phi_{t}+\zeta\left(k_{\text {out }}-k_{\text {in }}\right)|\nabla \phi|-\eta(g \kappa|\nabla \phi|+\nabla g \nabla \phi)=0 .
$$

For all the cases, the contour detector function $g$ is $e^{-\epsilon\left\|\nabla I_{\sigma}\right\|}$ with $\epsilon=10$ and $\sigma=0.8 \mathrm{~mm}$. The parameters $\zeta$ and $\eta$ are set equal 1.0. The Courant-FriedrichsLevy condition is used to compute the optimal time step for the equation. Less than twenty iterations are enough to assure convergence of the algorithm.

\section{Materials and Methods}

\subsection{Clinical and Computerized Protocol}

The study was performed on a data base of 39 brain aneurysms mainly located in the Circle of Willis. Image acquisition was performed using an Helical Elscint CT Twin scanner (Marconi; Haifa, Israel) with $120 \mathrm{kV} / 300 \mathrm{~mA}$ for the amplifier tube, $1.2-\mathrm{mm}$ collimation with an helical pitch of 1 and slice spacing of $0.65 \mathrm{~mm}$. Images were reconstructed on a 512 x 512 matrix with a square FOV of 20.8 $\mathrm{cm}$ yielding an in-plane resolution of $0.4 \mathrm{~mm}$. A total of $140 \mathrm{ml}$ of non ionic contrast fluid was intravenously administrated (Omnitrast $300 \mathrm{mg}$; Schering, Berling, Germany) at a rate of $3 \mathrm{ml} / \mathrm{s}$, starting the scanning 20 seconds after injection onset.

The manual quantification of the aneurysms was performed using 2D MIP images and measuring tools provided by the console software Omnipro (Marconi; Haifa, Israel). The clinical parameters needed for the planning of the endovascular intervention are the maximum neck diameter, the maximum width and maximum depth of the aneurysm. As it is customary in clinical routine, the measurements were carried out along several projection angles and from those, the neuroradiologist chose the view-angle producing maximal diameters.

Using the marching cubes algorithm, a 3D model of the aneurysm were reconstructed from the zero-level set of $\phi$. To make the computerized measurements comparable to the manual gold standard, the models were rendered with a viewpoint selected according to a similar criterion used to generate the MIP images. Two points were manually pin-pointed in the $3 \mathrm{D}$ scene corresponding to the ends of the measured magnitude in that angle. Measurements are then performed by projecting this points into the camera plane. 


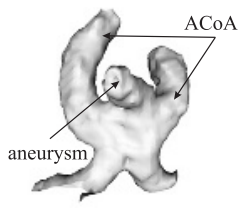

(a)

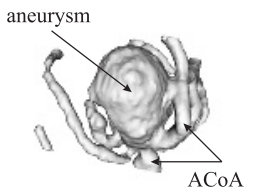

(b)

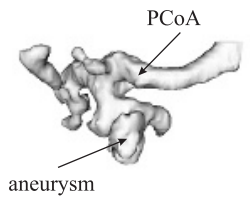

(c)

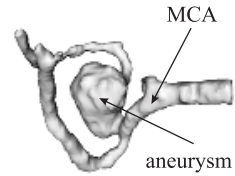

(d)

Fig. 3. Some representative examples of the models obtained by the algorithm. (a)(d) Anterior Communicating Artery, Giant Anterior Communicating Artery, Posterior Communicating Artery and Middle Cerebral Artery aneurysms, respectively.

\section{Results}

\subsection{Examples}

In Fig. 3 we show some examples of the segmentations of the most representative aneurysms presented in our data base.

\subsection{Evaluation}

Two experts carried out the manual measurements twice with enough delay between sessions to consider them independent. The average of the manual measurements is used as gold-standard and compared with the measurements obtained by the model based approach. Bland-Altman analysis 1 is used as statistical method to obtain the repeatability of the manual method for each of the two observers, the agreement between the observers and the agreement between the manual and the computerized method. The results of the study are shown in Table 1

Table 1. Results of the Bland-Altman analysis. The table shows mean $\pm \mathrm{SD}$ of the difference of the measurements in $\mathrm{mm}$. ObsI and ObsII stand for each observer and MB stands for the model-based technique.

\begin{tabular}{|c|cc|ccc|}
\hline \hline & ObsI & ObsII & \multicolumn{3}{|c|}{ ObsI vs ObsII ObsI vs MB ObsII vs MB } \\
\hline \hline Neck & $-0.07 \pm 1.09$ & $-0.51 \pm 0.86$ & $-0.03 \pm 1.22$ & $-0.47 \pm 1.05$ & $-0.44 \pm 0.91$ \\
Width & $0.94 \pm 1.87$ & $-0.34 \pm 1.35$ & $0.34 \pm 1.91$ & $0.23 \pm 1.86$ & $-0.11 \pm 1.43$ \\
Depth & $-0.65 \pm 2.41$ & $0.18 \pm 1.50$ & $-0.70 \pm 2.45$ & $-0.69 \pm 2.12$ & $0.00 \pm 1.55$ \\
\hline \hline
\end{tabular}

\section{Discussion and Conclusion}

Classic geodesic active contours approaches were unsatisfactory for segmenting the cerebral vasculature from CTA and more sophisticated speed functions in- 


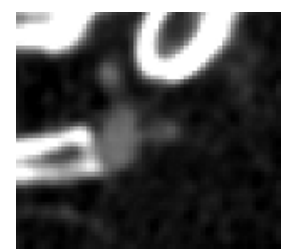

(a)

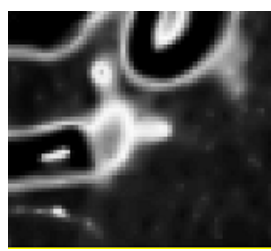

(b)

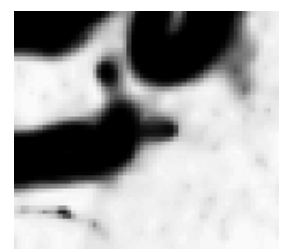

(c)

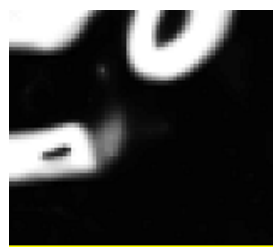

(d)

Fig. 4. Probability functions estimated by Gaussian models. Brighter areas correspond to higher probabilities. (a) Grey level image. (b)-(d) Probability for vessel, background and bone respectively.

troducing statistical information from the image were required to improve the classical approach. Most of the approaches found in the literature use Gaussian statistical information. Figure 4 shows an example of the tissue probability functions modelled by Gaussian distributions. Comparing with Figure 1, it can be appreciated that the probability of vessel is high in places of transition between bone and background. The probability of bone in the interior of the aneurysm is also high. Holes belonging to background inside the bone have high probability of being vessel. The introduction of these features in the region-based term, makes the model less robust and very sensitive to the parameters that have to be tuned for each patient to compensate for the effect of the misclassification. The use of non-parametric statistical information provides more accurate segmentations with minimal sensitivity for the selection of the parameters.

The features used by the $\mathrm{kNN}$ rule are computed at a single scale. It would seem that due to the nature of the object to be segmented, a multiscale approach should provide better results. However, it was observed that results were worse than using a single scale close to the voxel dimensions. This could be explained by the fact that as the number of scales increases, the dimensionality of the feature space also increases. This may deteriorate the performance of the classifier owing to the peaking phenomenon [8]. We are working on improvements on the classifier using a multiscale approach and dimensionality reduction strategies.

The aneurysms involved in the study had a mean size of $2.81 \mathrm{~mm}$ for the neck diameter, $5.40 \mathrm{~mm}$ for the width and $6.44 \mathrm{~mm}$ for the depth of the aneurysm with standard deviations of $0.84,2.95$ and $3.10 \mathrm{~mm}$ respectively. Results obtained with the manual method show that both observers have a similar performance in independent sessions. The repeatability study show a bias less than $1 \mathrm{~mm}$ in all the cases. The standard deviation is larger in the measurements of the aneurysm width and depth than in the neck diameter. The agreement study indicates also a bias less than $1 \mathrm{~mm}$ in all cases. The standard deviation is also larger in the measurement of the aneurysm width and depth than in the neck diameter. This could be logical as minimal variations in the selection of the view angle can yield large variations in the saccular dimensions of the aneurysm when measured on 
the images. These variations are less significative at the neck due to the smaller size of the measured magnitude and symmetry.

When comparing manual and computerized measurements, it can be observed that the bias is, in the worst case, approximately of the order of a voxel $(-0.69$ $\mathrm{mm})$. The standard deviations are lower in all the measurements. Therefore, the computerized method has a high agreement with each observer separately than the agreement achieved between the observers.

This study demonstrates the feasibility of using implicit deformable models combined with non-parametric statistical information to quantify aneurysm morphology and obtain clinically relevant parameters. In summary, the technique presented in this work will contribute to the computerized surgical planning of coiling procedures by allowing more accurate and truly $3 \mathrm{D}$ quantification of brain aneurysms.

Acknowledgments. The authors would like to acknowledge to Dr. R. Barrena and Dr. G. Hernandez from the Aragon Institute of Health Sciences (Zaragoza, Spain) for providing the data, measurements and clinical background. MHG is supported by the grant AP2001-1678 from the Spanish Ministry of Education, Culture and Sport. AFF is supported by a Ramon y Cajal Research Fellowship from the Spanish Ministry of Science and Technology (MCyT). GS is supported by ONI28NSF. This research was partially supported by the grants TIC20001635-C04-01 and TIC2002-04495-C02 from the MCyT.

\section{References}

1. J.M. Bland and D.G. Altman, Statistical methods for assessing agreement between two methods of clinical measurement, Lancet 8476 (1986 Feb), 307-10.

2. V. Caselles, R. Kimmel, and G. Sapiro, Geodesic active contours, International Journal of Computer Vision 22 (1) (1997), 61-79.

3. G.M. Debrun, V.A. Aletich, P. Kehrli, M. Misra, J.I. Ausman, F. Charbel, and H. Shownkeen, Aneurysm geometry: an important criterion in selecting patients for Guglielmi detachable coiling, Neuro. Med. Chir. 38 (1998 Apr), 1-20.

4. T. Deschamps, Extraction de courbes et surfaces par méthodes de chemins minimaux et ensembles de niveaux. Applications en imagerie medicale 3D, Ph.D. Thesis, University of Paris-Dauphine, France (2001 Dec).

5. A.F. Frangi, W.J. Niessen, P.D. Nederkoorn, J. Bakker, W.P.Th.M. Mali, and M.A. Viergever, Quantitative analysis of vessel morphology from 3D MR angiograms: in vitro and in vivo results, Mag. Res. in Med. 45(2) (2001 Feb), 311-22.

6. G. Guglielmi, F. Vinuela, I. Spetka, and V. Macellari, Electrothrombosis of saccular aneurysms via endovascular approach, Neruosurg. 75(1) (1991 Jul), 1-7.

7. M. Hernandez, G. Sapiro, and A.F. Frangi, Pre-clinical evaluation of implicit deformable models for three-dimensional segmentation of brain aneurysms in CTA, Proceedings of SPIE Medical Imaging, Image Processing 5032 (2003), 1264-74.

8. A.K. Jain and B. Chandrasekaran, Dimensionality and sample size considerations in pattern recognition practice, Handbook of Statistics 2 (1982), 835-855. 
9. L.M. Lorigo, O.D. Faugeras, W.E.L. Grimson, R. Keriven, R. Kikinis, A. Nabavi, and C.F. Westin., Curves: Curve evolution for vessel segmentation, Med. Im. Analysis $\mathbf{5 ( 3 )}$ (2001), 195-206.

10. S. Osher and J.A. Sethian, Fronts propagating with curvature-dependent speed: Algorithms based on Hamilton-Jacobi formulations, Journal of Computational Physics 79 (1988), 12-49.

11. N. Paragios, Geodesic active regions and level set methods: Contributions and applications in artificial vision, Ph.D. Thesis, University of Nice Sophia-Antipolis, France (2000).

12. P. Perona and J.Malik, Scale-space and edge detection using anisotropic diffusion, IEEE Trans. Patt. Anal. Machine Intel. 12(7) (1990), 629-639.

13. T.W. Raaymakers, G.J. Rinkel, M. Limburg, and A. Algra, Mortality and morbidity of surgery for unruptured intracranial aneurysms: a meta-analysis., Stroke 29(8) (1998 Aug), 1531-8.

14. M. Subasic, S. Loncaric, and E. Sorantin, 3-D deformable model segmentation of abdominal aneurysm, Proceedings of SPIE Medical Imaging, San Diego, USA, (2001).

15. P.C. Teo, G. Sapiro, and B. Wandell, Segmenting cortical gray matter for functional MRI visualization, 6th Int. Conf. Comp. Vis. (1998 Jan), 292-297.

16. A. Yezzi, A. Tsai, and A. Willsky, A statistical approach to snakes for bimodal and trimodal imagery, Computer Vision, 1999. The Proceedings of the Seventh IEEE International Conference on 2 (1999 Sept), 898-903. 